\title{
Ozaki Procedure
}

\author{
Ozaki Prosedürü \\ Cem Alhan (1) \\ Department of Cardiovascular Surgery, Acıbadem University, School of Medicine, İstanbul, Turkey
}

Numerous randomized-controlled (also controlled by the industry giants) trials have shown that, in terms of short-term outcomes, transcatheter aortic valve implantation (TAVI) offers a suitable alternative to the current gold standard of surgical aortic valve replacement (SAVR), even in low-risk patients. However, the main limitation of these trials is that the follow-up period is limited to 12 months alone ${ }^{[1,2]} \mathrm{In}$ a review of nearly 14,000 patients, five-year survival after TAVI was found to be $48 \% .^{[3]}$ Compared to the expected survival for a general population of similar age, this study demonstrated poorer survival outcomes after TAVI. In their recent meta-analysis comparing SAVR and TAVI in low- and intermediaterisk patients, Ueshima et al. ${ }^{[4]}$ demonstrated similar all-cause mortality, cardiac mortality, and neurological events during a two-year follow-up. Although the shortterm results have been well-studied in the literature, there is a limited number of data regarding long-term outcomes following TAVI, and with the unknown transcatheter valve durability, TAVI should not be offered to low-risk young patients, until long-term follow-up data are available.

Thus, SAVR is still the most optimal option for the great majority of patients who needs aortic valve replacement. In this video, the technical details of neocuspidization of the aortic valve (Ozaki procedure) will be presented.

\section{SURGICAL TECHNIQUE (Video)}

After a midline sternotomy, at least a $7 \times 8 \mathrm{~cm}$ pericardium should be harvested. Adipose tissue over the pericardium should be removed. Care must be taken not to damage the heart, phrenic nerve, and the pericardium, itself.

Pericardium is attached on the plate with a rough surface facing upwards. It must be stretched with threads to avoid shrinking during fixation.

Diaphragmatic side of the pericardium is marked as the larger leaflets would be made from this side, which is thicker.

The pericardium is soaked in $0.6 \%$ glutaraldehyde solution for $10 \mathrm{~min}$. It is, then, rinsed with saline for six min and this process is repeated three times.

A template of the pericardium is taken to close the pericardial defect at the end of the operation.

The aortic root is dissected free from the pulmonary artery. Aortotomy site is marked which must be $15 \mathrm{~mm}$ distal to the right coronary artery orifice.

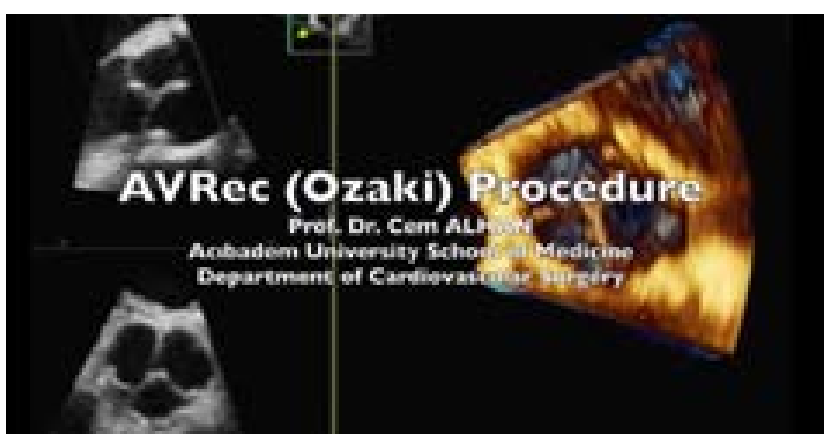

Video. Video showing all steps of Ozaki procedure.

Received: September 26, 2019 Accepted: September 30, 2019 Published online: October 23, 2019

Correspondence: Cem Alhan, MD. Acıbadem Maslak Hastanesi Kalp ve Damar Cerrahisi Bölümü, 34457 Maslak, istanbul, Turkey.

Tel: +90 542 - 5131947 e-mail: calhan@gmail.com 
A transverse aortotomy is made. The distance between the right end of the incision and the left coronary cusp (LCC)-non-coronary cusp (NCC) commissure must be $10 \mathrm{~mm}$.

For exposure, the distal aorta must be retracted with a pledgeted suture and the aortic retractors are placed.

Calcified aortic cusps are removed completely with maximal respect to the annular integrity. If necessary, an ultrasonic device may be used for decalcification.

Each intercommissural length is measured appropriately with the sizers. The midpoint of each annulus is marked.

Treated pericardium is placed on plate with a smooth surface facing upwards. The cusps are prepared according to the corresponding window on the template. Guiding dots are placed for every suture site.

Pericardium is attached on a piece of paper for easy handling during cutting. Each cusp is placed into the corresponding part of the petri dish filled with saline.

Suturing is started from the right coronary cusp which is most challenging. A $4-0,90 \mathrm{~cm}$ polypropylene suture with a TF $13 \mathrm{~mm}, 1 / 2$ circle needle is used for suturing. The first suture is passed from the midpoint of the cusp to the midpoint of the annulus. The cusp is dropped into the ventricle and the thread is tied with three knots.

Suturing is always from upside to downside on the annulus and vice versa on the cusp with a smooth surface of the cusp facing the left ventricle.

For the first three to four stictches on the annulus side, the distance between two stictches must be one third of the corresponding cusp distance, which gives the cusp a bird-nest shape. After this point where the remaining cusp and annulus lengths are the same, the distance between two stictches must be the same on both sides. The last stitch on the annulus must be a big bite for a better commissural support. After penetrating the last dot, the needle is straightened by the help of a needle-holder and passed $2.5 \mathrm{~mm}$ below the top of the commissure.

The other cusps are sutured in the same manner.

For the commissures, a $4-0,90 \mathrm{~cm}$ polypropylene suture with an RB-1 $17 \mathrm{~mm}, 1 / 2$ circle needle is used. This suture is placed at the midpoint between the free edge and the previous stitch (approx. $2.5 \mathrm{~mm}$ from edge) on one cusp and passed from the same site on the opposing cusp and, then, from the top corner of the wing, and lastly from the aorta. The other end of the suture is passed from the top corner of the other cusp and, then, the aorta. Thus, there remains four threads outside the aortic wall. A $5 \times 10 \mathrm{~mm}$ pledget is used to fix the threads to the aortic wall.

A good reconstruction must end in a "windmill" shape.

\section{Comment}

There are several advantages of the Ozaki procedure. First, there is no foreign body including a suture ring. It has been demonstrated that natural movement of the aortic annulus is preserved, and the reconstructed aortic valve has significantly less pressure gradients, compared to conventional aortic valve replacement cases due to maximal effective orifice area, which is particularly important in patients with a small aortic annulus. This technique can be also applied to almost all aortic valve pathologies. There is no need for warfarin postoperatively, and low-dose aspirin is used for three to six months.

On the other hand, the major drawback of the technique is the unknown durability. In a recent study, Ozaki et al. ${ }^{[5]}$ reported their experience in 850 consecutive patients operated for aortic stenosis, some of them with additional procedures (43.6\%). The median age was 71 years and the mean follow-up was $53.7 \pm 28.2$ months. The actuarial freedom from death, cumulative incidence of reoperation, and that of recurrent moderate-to-severe aortic regurgitation was $85.9 \%, 4.2 \%$, and $7.3 \%$, respectively, with the longest follow-up of 118 months. The authors reported no conversion to a prosthetic valve replacement, and only one of 850 patients underwent reoperation for structural valve degeneration.

\section{Declaration of conflicting interests}

The authors declared no conflicts of interest with respect to the authorship and/or publication of this article.

\section{Funding}

The authors received no financial support for the research and/or authorship of this article.

\section{REFERENCES}

1. Mack MJ, Leon MB, Thourani VH, Makkar R, Kodali SK, Russo M, et al. Transcatheter aortic-valve replacement with a balloon-expandable valve in low-risk patients. N Engl J Med 2019;380:1695-705.

2. Popma JJ, Deeb GM, Yakubov SJ, Mumtaz M, Gada H, O'Hair D, et al. Transcatheter aortic-valve replacement with a self-expanding valve in low-risk patients. N Engl J Med 2019;380:1706-15.

3. Chakos A, Wilson-Smith A, Arora S, Nguyen TC, Dhoble A, Tarantini G, et al. Long term outcomes of transcatheter 
aortic valve implantation (TAVI): a systematic review of 5-year survival and beyond. Ann Cardiothorac Surg 2017;6:432-43.

4. Ueshima D, Fovino LN, D’Amico G, Brener SJ, Esposito G, Tarantini G. Transcatheter versus surgical aortic valve replacement in low- and intermediate-risk patients: an updated systematic review and meta-analysis. Cardiovasc Interv Ther 2019;34:216-225

5. Ozaki S, Kawase I, Yamashita H, Uchida S, Takatoh M, Kiyohara N. Midterm outcomes after aortic valve neocuspidization with glutaraldehyde-treated autologous pericardium. J Thorac Cardiovasc Surg 2018;155:2379-87. 